Revue d'histoire de l'Amérique française

BEVUE D.HISTOIRE DE L'AMÉRIQUE FRANÇAISE

BENNETT, Margaret, Oatmeal and the Catechism. Scottish

Gaelic Settlers in Quebec (Edinburgh/Montréal, John Donald Publishers Ltd/McGill-Queen's University Press, 1998), 326 p.

\title{
Louise Cloutier
}

Volume 54, numéro 1, été 2000

URI : https://id.erudit.org/iderudit/305664ar

DOI : https://doi.org/10.7202/305664ar

Aller au sommaire du numéro

Éditeur(s)

Institut d'histoire de l'Amérique française

ISSN

0035-2357 (imprimé)

1492-1383 (numérique)

Découvrir la revue

Citer ce compte rendu

Cloutier, L. (2000). Compte rendu de [BENNETT, Margaret, Oatmeal and the

Catechism. Scottish Gaelic Settlers in Quebec (Edinburgh/Montréal, John Donald Publishers Ltd/McGill-Queen's University Press, 1998), 326 p.] Revue d'histoire de l'Amérique française, 54(1), 136-138. https://doi.org/10.7202/305664ar d'utilisation que vous pouvez consulter en ligne.

https://apropos.erudit.org/fr/usagers/politique-dutilisation/ 
BENNETT, Margaret, Oatmeal and the Catechism. Scottish Gaelic Settlers in Quebec (Edinburgh/

Montréal, John Donald Publishers Ltd/McGill-Queen's University Press, 1998), 326 p.

Margaret Bennett est née à Skye en Écosse dans l'une des îles de l'Archipel des Hébrides. Fille d'un ingénieur civil chargé de projets en énergie hydraulique à Lewis, elle vécut sur cette île de 1958 à 1964. Les Gaëls qui y habitent sont une branche des Celtes qui ont d'abord émigré en Irlande et puis, plus tard, en Écosse, au milieu du premier millénaire de notre ère. Le gaélique est et était la langue parlée par les habitants de ces îles situées au nord-ouest de l'Écosse. En 1972, doctorat en main, l'auteure de l'ouvrage Oatmeal and the Catechism. Scottish Gaelic Settlers in Quebec prit la décision d'étudier dans une des provinces de l'Est du Canada. Après quatre années d'études postdoctorales sur les traditions gaélo-écossaises à Terre-Neuve, elle accepta l'invitation du Musée national d'Ottawa (devenu Musée de la Civilisation) de travailler comme folkloriste, en collaboration avec deux anthropologues, au projet Québec-Hébrides. À la fin du projet de six semaines, il s'avéra que la recherche sur le terrain procura beaucoup plus de matériel que ne pouvait en récolter un folkloriste en si peu de temps. Cette première visite, complétée par une seconde en 1990 à Milan, dans les Cantons de l'Est, est à l'origine de cette publication. Margaret Bennett occupe actuellement le poste de chercheure honoraire associée au Centre d'études écossaises de l'Université de Glasgow et fait autorité dans le monde pour sa connaissance du folklore écossais.

L'ouvrage, divisé en neuf chapitres, pourrait se traduire ainsi: $D u$ Gruau et du catéchisme pour les immigrants gaélo-écossais au Québec. Cette recherche prend racine dans les Cantons de l'Est et couvre la période qui s'étend du milieu du XIx ${ }^{\mathrm{e}}$ siècle à nos jours. Profondément ancrée dans la tradition orale, l'œuvre embrasse les différentes disciplines qu'utilise le folklore, soit l'histoire, la linguistique, la géographie, la littérature, la sociologie, de même que l'étude des techniques de l'agriculture, de la chasse, de la pêche, de la botanique, de la médecine traditionnelle et de l'économie familiale. L'auteure aborde l'histoire de l'immigration, la vie domestique et communautaire, l'organisation religieuse et sociale, les coutumes et les croyances, le travail dévolu aux hommes et aux femmes, les événements de la vie culturelle (taigh-céilidh) et l'alimentation. Elle accorde une attention particulière aux chansons, aux poèmes, aux légendes et aux contes. 
Comment reconnaître un Écossais d'origine gaélique, sinon par son patronyme? L'auteure nous en fournit une liste impressionnante dans ses remerciements à Muriel Mayhew, Duncan McLeod, Ruth Nicolson ainsi qu'aux MacArthur, MacDonald, Maclver, Mackenzie, MacRae, Matheson, Morrison, Murray, Nicolson, Sherman, Smith et Young, pour n'en citer que quelques-uns.

Parler de gruau (oatmeal), c'est parler symboliquement de survie, c'est rappeler la difficulté de vivre, durant la première année de leur arrivée, des immigrants hébridiens écossais de langue gaélique, venus d'aussi loin que des îles Lewis, Harris, Skye et Arran afin d'améliorer leur sort. Graduellement, il en résulta une vie plus harmonieuse et plus confortable grâce au courage, à l'esprit d'invention et à la hardiesse de ces immigrants devenus Québécois.

La religion fut d'un grand secours pour la communauté d'alors, réunie autour du ministre de l'Église écossaise libre (Free Scottish Church), respectueuse du rituel presbytérien né de la Réforme. La langue gaélique était parlée par tous dès leur arrivée. Par la suite, cependant, comme l'indiquent les statistiques du recensement des Cantons de l'Est au Québec entre 1851 et 1891, l'anglais et le français s'infiltrent dans ces communautés, quoique de façon très inégale d'un village à l'autre, comme le montre le tableau suivant:

\begin{tabular}{llll}
\hline Villages & Gaélique & Français & Anglais \\
\hline Marsboro & c. $75 \%$ & c. $20 \%$ & c. $5 \%$ \\
Milan & c. $95 \%$ & c. $5 \%$ & c. $0 \%$ \\
Scotstown & c. $50 \%$ & c. $25 \%$ & c. $25 \%$ \\
Springhill & c. $50 \%$ & c. $25 \%$ & c. $25 \%$ \\
Stornoway & c. $95 \%$ & c. $3 \%$ & c. $2 \%$ \\
Red Mountain & c. $75 \%$ & c. $20 \%$ & c. $5 \%$ \\
\hline
\end{tabular}

Au début des années 1970, la langue gaélique est menacée d'extinction par la langue anglaise. Et au tournant du xxi ${ }^{e}$ siècle, le français québécois prédomine.

Cet ouvrage est très utile et suscite notre admiration pour la transcription des conversations enregistrées en anglais et en gaélique et pour le traitement du contenu. Une bibliographie étoffée, des cartes et des photos d'époque viennent appuyer le texte. La présence d'une communauté gaélique ne fait plus de doute. La région délimitée est si bien 
circonscrite qu'elle a le défaut de laisser croire en l'absence de Gaëls dans d'autres régions du Québec. La reconnaissance et la répartition des pratiques traditionnelles entre Écossais, Canadiens français et Amérindiens mérite attention. Nulle part il n'est fait mention de traditions anglaises. L'apport économique et culturel de cette minorité est remarquable. Notons toutefois que la politique ne fait pas partie de la liste des thèmes traités.

Ce travail soulève une problématique complexe et paradoxale. Lorsqu'on perd sa langue, perd-on sa culture? Pour en comprendre la complexité, un peu d'histoire s'impose.

Tout lecteur averti comprendra à quel point la "Auld Alliance" France-Écosse qui avait été forte, étroite et cordiale, dès le $\mathrm{Ix}^{\mathrm{e}}$ siècle en Europe, se répercuta dans la nouvelle colonie anglaise du Canada, après 1759. Si l'Écosse avait été conquise par les troupes anglaises, à la bataille de Culloden, en 1746, il n'en restait pas moins que ses habitants, bien que sujets britanniques, demeuraient des Gaëls d'Écosse (Highlanders et Islanders), d'une part et des Anglo-Normands d'Écosse (Lowlanders), d'autre part.

Si les Écossais ne sont pas des Anglais, si les conquérants anglais les ont envoyés s'établir au Québec, de qui ou de quoi parle-t-on quand on parle des Anglais à la frontière du Québec et de l'Ontario? 